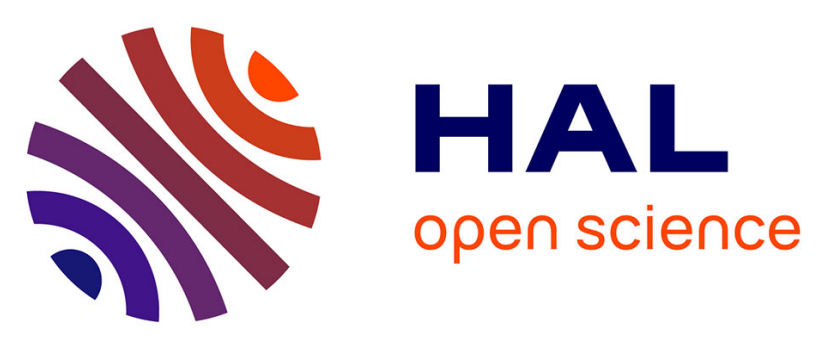

\title{
Nonlinear control of the longitudinal rotational dynamics of a flexible aircraft
}

Elodie Duraffourg, Laurent Burlion, Tarek Ahmed-Ali, Françoise

Lamnabhi-Lagarrigue

\section{- To cite this version:}

Elodie Duraffourg, Laurent Burlion, Tarek Ahmed-Ali, Françoise Lamnabhi-Lagarrigue. Nonlinear control of the longitudinal rotational dynamics of a flexible aircraft. 12th European Control Conference (ECC 2013), Jul 2013, Zürich, Switzerland. pp.335-340, 10.23919/ECC.2013.6669369 hal-00826145

\section{HAL Id: hal-00826145 \\ https://hal-centralesupelec.archives-ouvertes.fr/hal-00826145}

Submitted on 17 Jan 2022

HAL is a multi-disciplinary open access archive for the deposit and dissemination of scientific research documents, whether they are published or not. The documents may come from teaching and research institutions in France or abroad, or from public or private research centers.
L'archive ouverte pluridisciplinaire HAL, est destinée au dépôt et à la diffusion de documents scientifiques de niveau recherche, publiés ou non, émanant des établissements d'enseignement et de recherche français ou étrangers, des laboratoires publics ou privés.

\section{다)(1) $(5$}

Distributed under a Creative Commons Attribution - NonCommerciall 4.0 International 


\title{
Non-linear control of the longitudinal rotational dynamics of a flexible aircraft
}

\author{
Elodie Duraffourg ${ }^{1}$, Laurent Burlion ${ }^{1}$, Tarek Ahmed-Ali ${ }^{2}$ and Françoise Lamnabhi-Lagarrigue ${ }^{3}$
}

\begin{abstract}
This paper studies the longitudinal inner loop control design of a flexible aircraft over a large flight domain. More precisely, the objective is to construct a stabilizing flight control law to steer the angle of attack to a desired trim value while suppressing the oscillation of the bending modes. A new Lyapunov-based non-linear controller is designed to enhance the transient of the flexible modes before reaching the zero dynamics sub-manifold. Moreover, the local behavior of the trajectories is finally modified by blending our controller with a LQ controller in order to achieve the additional and practical control objective of locally damping out the oscillations of the bending modes.
\end{abstract}

\section{INTRODUCTION}

The problem of designing a semi-global or global asymptotic controller for a non-linear system of the following form

$$
\left\{\begin{aligned}
\dot{x} & =f(x)+g(x) u \\
\dot{z} & =f_{z}(z, x)
\end{aligned}\right.
$$

where $f_{z}(0,0)=0$, the dynamics of $z \in \mathbb{R}^{r}$ is called the zero dynamics (when $x=0$ ), the dynamics of $x \in \mathbb{R}^{n}$ is upper triangular and $u \in \mathbb{R}$ is a challenging problem in the field of non-linear control. Indeed, it is well known that when the functions $f$ and $g$ are perfectly known the $x$-dynamics can be transformed to a chain of integrators and the system is in normal form [6]. Otherwise, the $x$-dynamics can be controlled by a Backstepping controller [7] but in both cases the work is not finished and two kind of problems arise depending on the stability of the zero-dynamics.

The 'most difficult' problem is surely the case of non minimum phase non-linear systems for which there has been a lot of advances in the last few years (see [8], [9], [10], [12], [13] to cite a few). Although many results have been given for some sub-classes of systems and many applications have been proposed, it seems to us that the problem is still open. Indeed, to the best of our knowledge, when (obviously) $f_{z}$ is not linear with respect to some variables, there is a lack of theoretical results when there are more than two components of $x$ in $f_{z}$ (see [9]). Moreover, most of the applications considered have a one or two-dimensional zero dynamics and it does not seem obvious to extend the results to larger classes of systems.

1 E. Duraffourg and L. Burlion are with Onera - The French Aerospace Lab, F-31055 Toulouse, France. Elodie.Duraffourg (at) onera.fr Laurent.Burlion (at) onera.fr

${ }^{2}$ T. Ahmed-Ali is with Ecole Nationale Supérieure d'Ingénieurs de Caen (ENSICAEN), 6 boulevard du Marchal Juin 14050 Caen Cedex 4, France. tarek.ahmed-ali (at) greyc.ensicaen.fr

3 F. Lamnabhi-Lagarrigue is with L2S-CNRS, Supélec, 3 rue Joliot Curie, 91192 Gif-sur-Yvette, France. lamnabhi (at) lss.supelec.fr
Concerning the problem of minimum phase non-linear systems, there is still no general global stabilization result when we relax the assumptions of $f_{z}$ although the problem is apparently less difficult and has been early solved by simply stabilizing the $x$-dynamics by using a controller of the form $u=u(x)$ under some hypothesis on the growth of $f_{z}$. Indeed, it is known that the available result is only semi-global when $f_{z}(z, x)$ depends only on one component of $x_{[2, n]}:=[x(2), \ldots, x(n)]$. The interested reader will find more details in [6] and [11] where a controller $u=u(x, z)$ is proposed and globally stabilizes some special classes of minimum phase non-linear systems.

Our interpretation of this result is that a controller which depends on $z$ has led to a better result because it considered the full state during the transient.

In this paper, we are interested in stabilizing the rotational dynamics of a flexible aircraft (which belongs to the class of single-input minimum phase non-linear systems) by using a controller which depends on $z$ too: our aim is to both enhance the transient of the full state and the local behavior of the closed loop system.

In our application, $z$ is the vector formed by the flexible modes and their derivatives. By using $z$ in a controller which is inspired by some recent papers [3], [4], we show that we obtain a better transient of the flexible modes when the flexible frequencies are within the bandwidth of the controller i.e when there is a strong coupling between the rigid and flexible modes (to highlight our results, the model of the flexible airplane has been inspired from [5], [2] but the flexible modes pulsations are quite low). Moreover, in this paper, we also propose to study the local behavior of the closed-loop system in the case where the damping of the flexible modes are very low. To solve the problem of damping out these modes, we propose to unite our controller with an optimal LQ controller in the vicinity of the origin: we then use the results of [1] in order to guarantee the stability of this hybrid system.

The paper is organized as follows: in section II a theoretical result is given which uses and extends the results of [3] to a broader class of system thanks to the use of a modified Lyapunov function. Thanks to this extension and after a change of coordinates, this result is applied to the rotational dynamics of the flexible aircraft in section III. Section IV presents a method to damp out the oscillations of the bending modes. We finally give our conclusions and some future research directions. 


\section{A THEORETICAL RESULT ON THE LYAPUNOV BASED CONTROLLER DESIGN}

This section formulates a theoretical result which is used in section III to construct a stabilizing controller (of the form $u=u(x, \mathbf{z}))$ for the rotational dynamics of a flexible aircraft.

\section{A. System under consideration and objectives}

All along this section, the following non-linear system is considered:

$$
\left\{\begin{aligned}
\dot{x} & =u \\
\dot{z} & =f_{z}(z, x, u):=A_{z} z+F(x) x+H(x) u
\end{aligned}\right.
$$

where $(x, z) \in \mathbb{R} \times \mathbb{R}^{n-1}$ is the state, $u$ in $\mathbb{R}$ is the control input, $f_{z}$ is Input to State Stable (ISS) with respect to the inputs $x$ and $u, H: \mathbb{R} \rightarrow \mathbb{R}^{n-1}$ and $F: \mathbb{R} \rightarrow \mathbb{R}^{n-1}$ are $C^{1}$ functions, and $A_{z} \in \mathbb{R}^{n-1 \times n-1}$ is Hurwitz. The dynamics of $z$ is called the 'flexible dynamics'. $A_{z}$ being Hurwitz, there exist two symmetric positive definite matrices $P_{z}$ and $Q_{z}$ in $\mathbb{R}^{n-1 \times n-1}$ such that

$$
A_{z}^{T} P_{z}+P_{z} A_{z}=-2 Q_{z}
$$

The objective is to design a globally stabilizing controller to steer the state $x$ of system (2) from an initial value to a desired equilibrium referred to as $x_{c}$. Thus the errors are defined as $\delta x=x-x_{c}, \delta z=z-z_{c}$, with $z_{c}=-A_{z}^{-1} F\left(x_{c}\right) x_{c}$ and system (2) is turned into:

$$
\left\{\begin{array}{l}
\dot{\delta} x=u \\
\dot{\delta} z=A_{z} \delta z+\bar{F}(x) \delta x+H(x) u
\end{array}\right.
$$

where $\bar{F}(x)=F(x) x-F\left(x_{c}\right) x_{c}{ }^{1}$.

Please note that this 'simple' class (with respect to the $x$ dynamics) has been chosen to suit our applicative part and that the control design proposed in this section could be used for a larger class of systems where $x \in \mathbb{R}^{n_{x}>1}$ and where the $x$-dynamics is upper-triangular.

\section{B. Non-linear control design}

A new Lyapunov-based controller inspired by [3] is designed to achieve the control objectives.

Assumption 1: There exists a positive real $H_{M}$ such that:

$$
\forall x \in \mathbb{R} \quad\|H(x)\|_{2} \leq H_{M}
$$

Thanks to Assumption 1, the integral of $H$ is well-defined and the following Lyapunov function candidate can be considered for system (4)

$$
V=\frac{c_{1}}{2} \delta x^{2}+\frac{c_{2}}{2}\left[\delta z^{T} P_{z} \delta z-2 \delta z^{T} P_{z} \int_{x_{c}}^{x} H(s) d s\right]
$$

${ }^{1} \bar{F}(x)$ is computed as follow:

$$
\begin{aligned}
& \bar{F}(x)=\frac{F(x) x-F\left(x_{c}\right) x_{c}}{x-x_{c}} \text { if } x \neq x_{c} \\
& \bar{F}(x)=\frac{d(F(x) x)}{d x} \text { if } x=x_{c}
\end{aligned}
$$

where $c_{1}, c_{2}$ are positive constants. Throughout this paper, we use the following notation:

$$
\mathscr{H}(x) \delta x:=\int_{x_{c}}^{x} H(s) d s
$$

Assumption 2: $c_{1}$ and $c_{2}$ are defined such that:

$$
c_{2}<\frac{c_{1} \lambda_{\min }\left(P_{z}\right)}{H_{M}^{2} \lambda_{\max }\left(P_{z}^{2}\right)}
$$

where $\lambda_{\min }(A)$ represents the smallest eigenvalue of matrix $A$ and $\lambda_{\max }(A)$ the highest one.

Proposition 1: Under Assumptions 1 and 2, the function $V$ is positive definite.

Proof 1: Assumption 1 yields $\|\mathscr{H}(x)\|_{2} \leq H_{M}$ and

$$
V \geq \frac{c_{1}}{2} \delta x^{2}+\frac{c_{2}}{2}\left[\delta z^{T} P_{z} \delta z-2\left|\delta z^{T} P_{z} H_{M} \delta x\right|\right]
$$

For all $k>0$ we have:

$$
-2\left|\delta z^{T} P_{z} H_{M} \delta x\right| \geq-k^{2} \delta x^{2}-\frac{H_{M}^{2}}{k^{2}} \delta z^{T} P_{z}^{2} \delta z
$$

Consequently the function $V$ satisfies:

$$
V \geq \frac{1}{2}\left(c_{1}-k^{2} c_{2}\right) \delta x^{2}+\frac{c_{2}}{2} \delta z^{T}\left(P_{z}-\frac{H_{M}^{2}}{k^{2}} P_{z}^{2}\right) \delta z
$$

$V$ is positive definite if: $c_{1}>k^{2} c_{2}$ and $P_{z}>\frac{H_{M}^{2}}{k^{2}} P_{z}^{2}$. Since we have: $P_{z}>\lambda_{\min }\left(P_{z}\right) I_{n-1}$ and $\lambda_{\max }\left(P_{z}^{2}\right) I_{n-1}>P_{z}^{2}$, with $I_{n-1}$ the identity matrix, the condition $P_{z}>\frac{H_{M}^{2}}{k^{2}} P_{z}^{2}$ is verified if:

$$
\lambda_{\min }\left(P_{z}\right) \geq \frac{H_{M}^{2}}{k^{2}} \lambda_{\max }\left(P_{z}^{2}\right)
$$

We finally choose: $k^{2}=H_{M}^{2} \frac{\lambda_{\max }\left(P_{z}^{2}\right)}{\lambda_{\min }\left(P_{z}\right)}$ to get the result.

Proposition 2: Consider the system (2), with the control law:

$$
u(x, z)=-\frac{\lambda_{x} \delta x+\Gamma(x, z)}{\Delta(x)}
$$

with $\lambda_{x}>0$ and:

$$
\begin{aligned}
\Gamma(x, z) & =c_{2} \delta z^{T} P_{z} \bar{F}(x)-c_{2}\left(A_{z} \delta z+\bar{F}(x) \delta x\right)^{T} P_{z} \mathscr{H}(x) \\
\Delta(x) & =c_{1}-c_{2} H(x)^{T} P_{z} \mathscr{H}(x)
\end{aligned}
$$

Under Assumptions 1 and 2, the closed loop system is globally and asymptotically stable.

Proof 2: The time derivative of $V$ is:

$$
\dot{V}=\delta x[u \Delta(x)+\Gamma(x, z)]-c_{2} \delta z^{T} Q_{z} \delta z
$$

Using assumption 2 and the fact that $P_{z}$ is symmetric yield:

$$
c_{1}>\frac{c_{2} H_{M}^{2} \lambda_{\max }\left(P_{z}\right) \lambda_{\max }\left(P_{z}\right)}{\lambda_{\min }\left(P_{z}\right)} \geq c_{2} H_{M}^{2} \lambda_{\max }\left(P_{z}\right)
$$


Then the function $\Delta$ satisfies $\Delta(x) \geq c_{1}-c_{2} H_{M}^{2} \lambda_{\max }\left(P_{z}\right)>0$ and the control law (9) is well-defined. The time derivative of $V$ is finally given by:

$$
\dot{V}=-\lambda_{x} \delta x^{2}-c_{2} \delta z^{T} Q_{z} \delta z
$$

which satisfies $\dot{V}<0$. Lyapunov stability theorem proves global and asymptotic stability of the origin of the closed loop composed of system (2) and control law (9).

This result deserves a few additional comments:

- Remark 1: By comparison to a law of the form $u=-\tilde{\lambda}_{x} x$ which would stabilize $x$ and render the whole system stable (since the zero dynamics is minimum phase and $f_{z}$ is ISS), the control law (9) uses the flexible dynamics $z$ and should limit the interaction of the $x$-dynamics on the transient of the $z$-dynamics. This fact will be illustrated on our application in the next section.

- Remark 2: As opposed to [3], our Lyapunov function uses an integral term: this is due to the non-linear gain $H(x)$ of the control law $u$ in the flexible dynamics. This integral enabled us to relax the assumptions on the function $H$.

\section{APPLICATION TO THE ROTATIONAL DYNAMICS OF A FLEXIBLE AIRCRAFT}

The model of the longitudinal dynamics of a flexible hypersonic vehicle is given in [2]. Inspired by this model that was first-developed in [5], the rotational dynamics, namely the angle of attack $\alpha$ and pitch rate $q$, and the flexible dynamics are considered in this study. The equations of motion are written as:

$$
\left\{\begin{array}{l}
\dot{\alpha}=f_{0}(\alpha) \alpha+C_{\alpha}^{\eta_{1}} \eta_{1}+C_{\alpha}^{\eta_{2}} \eta_{2}+q+C_{\alpha}^{c} \\
\dot{q}=f_{1}(\alpha) \alpha+C_{q}^{\eta_{1}} \eta_{1}+C_{q}^{\eta_{2}} \eta_{2}+C^{v} v \\
\ddot{\eta}_{1}=h_{1} v-\omega_{1}^{2} \eta_{1}-2 \xi_{1} \omega_{1} \dot{\eta}_{1}+N_{1}^{\eta} \eta_{2}+f_{\eta_{1}}(\alpha) \alpha \\
\ddot{\eta}_{2}=h_{2} v-\omega_{2}^{2} \eta_{2}-2 \xi_{2} \omega_{2} \dot{\eta}_{2}+N_{2}^{\eta} \eta_{1}+f_{\eta_{2}}(\alpha) \alpha
\end{array}\right.
$$

The flexible aircraft dynamics (12) is composed of two rigid body state variables $[\alpha, q]^{T}$ and four flexible states $z=\left[\eta_{1}, \dot{\eta}_{1}, \eta_{2}, \dot{\eta}_{2}\right]^{T}$ corresponding to the first two bending modes. The interaction between rigid and flexible dynamics is also considered. The control input is $v . f_{0}, f_{1}, f_{\eta_{1}}$ and $f_{\eta_{2}}$ are real functions of class $C^{1}$. Moreover $f_{0}$ is bounded.

We assume that the entire state $\left[\alpha, q, z^{T}\right]^{T}$ is measured and the system is initially at a trim flight condition. The objective is to design a non-linear controller to steer the angle of attack from an initial equilibrium to desired trim condition $\alpha_{c}$, while suppressing the oscillations of the bending modes. $\alpha_{c}$ and the initial angle of attack trim are supposed to be in an admissible range, referred to as $\mathscr{A}$.

\section{A. Transformation of the system}

The first step consists in transforming the system (12) to fit with the theory. That is made using the following change of coordinates:

$$
\begin{aligned}
q^{*} & =f_{0}(\alpha) \alpha+C_{\alpha}^{\eta_{1}} \eta_{1}+C_{\alpha}^{\eta_{2}} \eta_{2}+q+C_{\alpha}^{c} \\
\bar{\eta}_{i} & =\eta_{i}-\frac{h_{i}}{C^{u}} \alpha, \quad i \in 1,2
\end{aligned}
$$

Indeed, substituting (13) into the first equation of (12) and (14) into the flexible dynamics of (12) yields

$$
\begin{aligned}
\dot{\alpha} & =q^{*} \\
\dot{\bar{z}} & =A_{z} \bar{z}+F(\alpha) \alpha+H(\alpha) q^{*} \\
\dot{q}^{*} & =\Lambda\left(q^{*}, \alpha, \bar{z}\right)+C^{v} \delta v
\end{aligned}
$$

with the following notations:

$$
\begin{gathered}
\bar{z}=\left[\begin{array}{llll}
\bar{\eta}_{1} & \dot{\bar{\eta}}_{1} & \bar{\eta}_{2} & \dot{\bar{\eta}}_{2}
\end{array}\right]^{T} \\
A_{z}=\left[\begin{array}{cccc}
0 & 1 & 0 & 0 \\
-\bar{\omega}_{1}^{2} & -2 \bar{\xi}_{1} \bar{\omega}_{1} & \bar{N}_{1}^{\eta} & \bar{N}_{1}^{\dot{\eta}} \\
0 & 0 & 0 & 1 \\
\bar{N}_{2}^{\eta} & \bar{N}_{2}^{\dot{\eta}} & -\bar{\omega}_{2}^{2} & -2 \bar{\xi}_{2} \bar{\omega}_{2}
\end{array}\right] \\
H(\alpha)=\left[\begin{array}{llll}
0 & f_{q}^{1}(\alpha) & 0 & f_{q}^{2}(\alpha)
\end{array}\right]^{T} \\
F(\alpha)=\left[\begin{array}{llll}
0 & f_{\alpha}^{1}(\alpha) & 0 & f_{\alpha}^{2}(\alpha)
\end{array}\right]^{T} \\
\left(\begin{array}{c}
\bar{N}_{1}^{\eta} \\
\bar{N}_{2}^{\eta}
\end{array}\right)=\left(\begin{array}{l}
N_{1}^{\eta} \\
N_{2}^{\eta}
\end{array}\right)-\frac{1}{C^{v}}\left(\begin{array}{cc}
0 & h_{1} \\
h_{2} & 0
\end{array}\right)\left(\begin{array}{l}
C_{q}^{\eta_{1}} \\
C_{q}^{\eta_{2}}
\end{array}\right) \\
\left(\begin{array}{l}
\bar{N}_{1}^{\dot{\eta}} \\
\bar{N}_{2}^{\dot{\eta}}
\end{array}\right)=-\frac{1}{C^{v}}\left(\begin{array}{cc}
0 & h_{1} \\
h_{2} & 0
\end{array}\right)\left(\begin{array}{l}
C_{\alpha}^{\eta_{1}} \\
C_{\alpha}^{\eta_{2}}
\end{array}\right)
\end{gathered}
$$

$\forall i \in\{1,2\}, \bar{\omega}_{i}^{2}=\omega_{i}^{2}+\frac{h_{i}}{C^{v}} C_{q}^{\eta_{i}}, \quad 2 \bar{\xi}_{i} \bar{\omega}_{i}=2 \xi_{i} \omega_{i}+\frac{h_{i}}{C^{v}} C_{\alpha}^{\eta_{i}}$ $f_{q^{*}}^{i}(\alpha)=-\frac{h_{i}}{C^{v}}\left(\frac{d f_{0}(\alpha)}{d \alpha} \alpha+f_{0}(\alpha)+C_{\alpha}^{\eta_{1}} \frac{h_{1}}{C^{v}}+C_{\alpha}^{\eta_{2}} \frac{h_{2}}{C^{v}}+2 \xi_{i} \omega_{i}\right)$ $\left(\begin{array}{l}f_{\alpha}^{1}(\alpha) \\ f_{\alpha}^{2}(\alpha)\end{array}\right)=\left(\begin{array}{l}f_{\eta_{1}}(\alpha) \\ f_{\eta_{2}}(\alpha)\end{array}\right)-\frac{1}{C^{v}}\left(\begin{array}{cc}g(\alpha)+\omega_{1}^{2} & -N_{1}^{\eta} \\ -N_{2}^{\eta} & g(\alpha)+\omega_{2}^{2}\end{array}\right)\left(\begin{array}{l}h_{1} \\ h_{2}\end{array}\right)$ $g(\alpha)=f_{1}(\alpha)+C_{q}^{\eta_{1}} \frac{h_{1}}{C^{v}}+C_{q}^{\eta_{2}} \frac{h_{2}}{C^{v}}$

$$
\begin{gathered}
\Lambda\left(q^{*}, \alpha, \bar{z}\right)=q^{*}\left[\frac{d f_{0}(\alpha)}{d \alpha} \alpha+f_{0}(\alpha)+C_{\alpha}^{\eta_{1}} \frac{h_{1}}{C^{v}}+C_{\alpha}^{\eta_{2}} \frac{h_{2}}{C^{v}}\right] \\
+\bar{z}^{T} M+\alpha\left[f_{1}(\alpha)+C_{q}^{\eta_{1}} \frac{h_{1}}{C^{v}}+C_{q}^{\eta_{2}} \frac{h_{2}}{C^{v}}\right] \\
M=\left[C_{q}^{\eta_{1}} C_{\alpha}^{\eta_{1}} C_{q}^{\eta_{2}} C_{\alpha}^{\eta_{2}}\right]^{T}
\end{gathered}
$$

The trim state is then given by: $\left[\alpha_{c}, q_{c}, z_{c}^{T}\right]^{T}$ where: $q_{c}=0$ and $z_{c}=\left[\eta_{1}^{c}, 0, \eta_{2}^{c}, 0\right]^{T}$ with:

$$
\left(\begin{array}{cc}
\bar{\omega}_{1}^{2} & -\bar{N}_{1}^{\eta} \\
-\bar{N}_{2}^{\eta} & \bar{\omega}_{2}^{2}
\end{array}\right)\left(\begin{array}{l}
\eta_{1}^{c} \\
\eta_{2}^{c}
\end{array}\right)=\alpha_{c}\left(\begin{array}{c}
f_{\eta_{1}}\left(\alpha_{c}\right)-\frac{h_{1}}{C^{v}} f_{1}\left(\alpha_{c}\right) \\
f_{\eta_{2}}\left(\alpha_{c}\right)-\frac{h_{2}}{C^{v}} f_{1}\left(\alpha_{c}\right)
\end{array}\right)
$$

and the trim input is $v_{c}=-\frac{1}{C^{v}}\left(f_{1}\left(\alpha_{c}\right) \alpha_{c}+C_{q}^{\eta_{1}} \eta_{1}^{c}+C_{q}^{\eta_{2}} \eta_{2}^{c}\right)$.

Sub-system (15)-(16) belongs to the class of system defined in section II, by taking $x=\alpha$ and $u=q^{*}$. Consequently the theoretical result can be applied to this system.

\section{B. Application of proposition 2}

Before applying this result, the assumptions have to be checked.

Since the objective is to make a change of the trim conditions, $\alpha(0)$ and $\alpha_{c}$ are both in $\mathscr{A}$ and if we succeed to build a decreasing Lyapunov function $V$ for the whole system then $|\alpha|$ will remain bounded by $\left|\alpha_{c}\right|+\left|V^{-1}(\delta \alpha(t=0), 0)\right|$. 
And thus $f_{q^{*}}^{i}$ is bounded. Consequently, for all $\alpha(0)$ and $\alpha_{c}$ in $\mathscr{A}$, there exists positive constants $f_{m}^{i}$ such that:

$$
\left|f_{q^{*}}^{i}\right| \leq f_{m}^{i}, i \in\{1,2\}
$$

And $\|H(\alpha)\|_{2}=\sqrt{\left|f_{q^{*}}^{1}\right|^{2}+\left|f_{q^{*}}^{2}\right|^{2}} \leq \sqrt{f_{m}^{1^{2}+f_{m}^{2}}}$. So $H_{M}$ exists and assumption 1 is true.

Assumption 2 is guaranteed by choosing: $c_{2}<c_{2 \max }$ with:

$$
c_{2 \max }=\frac{c_{1}}{H_{M}^{2}} \frac{\lambda_{\min }\left(P_{z}\right)}{\lambda_{\max }\left(P_{z}\right)}
$$

Proposition 2 can finally be applied to sub-system (15)(16). A control law, denoted $q_{\mathrm{cmd}}^{*}$, given by the application of (9) to sub-system (15)-(16) is then available and actually corresponds to an intermediate virtual control command for sub-system (15)-(16).

\section{Control of the whole system}

An additional step of Backstepping is necessary to control the whole system through control input $v$. Consider the following Lyapunov function candidate for system (15)-(16)(17)

$$
V_{\infty}=V(\delta \alpha, \delta \bar{z})+\frac{c_{3}}{2} \delta q^{* 2}
$$

with $c_{3}$ a positive constant, $\delta q^{*}=q^{*}-q_{\mathrm{cmd}}^{*}$ and $V$ the Lyapunov function (6) applied to sub-system (15)-(16). Assumptions 1 being true, function $V_{\infty}$ is positive definite. The derivative of $V_{\infty}$ is given by:

$$
\begin{array}{r}
\dot{V}_{\infty}=\delta q^{*}\left[\delta \alpha \Delta(\alpha)+c_{3}\left(\Lambda\left(q^{*}, \alpha, \bar{z}\right)+C^{v} \delta v-\dot{q}_{\mathrm{cmd}}^{*}\right)\right] \\
-c_{2} \delta \bar{z}^{T} Q_{z} \delta \bar{z}-\lambda_{\alpha} \delta \alpha^{2}
\end{array}
$$

Consider the feedback control law:

$$
\delta v=\frac{1}{C^{v}}\left[\dot{q}_{\mathrm{cmd}}^{*}-\Lambda\left(q^{*}, \alpha, \bar{z}\right)-\frac{1}{c_{3}}\left(\delta \alpha \Delta(\alpha)+\lambda_{q} \delta q^{*}\right)\right]
$$

The expression of $\dot{V}_{\infty}$ is:

$$
\dot{V}_{\infty}=-\lambda_{\alpha} \delta \alpha^{2}-\lambda_{q} \delta q^{* 2}-c_{2} \delta \bar{z}^{T} Q_{z} \delta \bar{z}
$$

with $\lambda_{q}>0$ which satisfies $\dot{V}_{\infty}<0$ and proves the stability of the equilibrium of the closed loop system (12).

Note that in this application the stability proof requires the initial trim to be in $\mathscr{A}$.

\section{Simulation results}

The controller has been tested with:

$$
\begin{aligned}
f_{0}(\alpha) & =C_{\alpha}^{2} \sin (\alpha)+C_{\alpha}^{1} \\
f_{1}(\alpha) & =C_{q}^{2} \alpha+C_{q}^{1} \\
f_{\eta_{1}}(\alpha) & =N_{\eta_{1}}^{2} \alpha+N_{\eta_{1}}^{1} \\
f_{\eta_{2}}(\alpha) & =N_{\eta_{2}}^{2} \alpha+N_{\eta_{2}}^{1}
\end{aligned}
$$

The damping of the bending modes is chosen equal to 0.01 . The aircraft is initially trimmed with $\alpha(0)=-10^{\circ}$ and the admissible set for $\alpha$ ranged from $-35^{\circ}$ to $35^{\circ}$. At time equals to $1 \mathrm{~s}$, trim conditions are changed and desired angle of attack is $\alpha_{c}=25^{\circ}$.
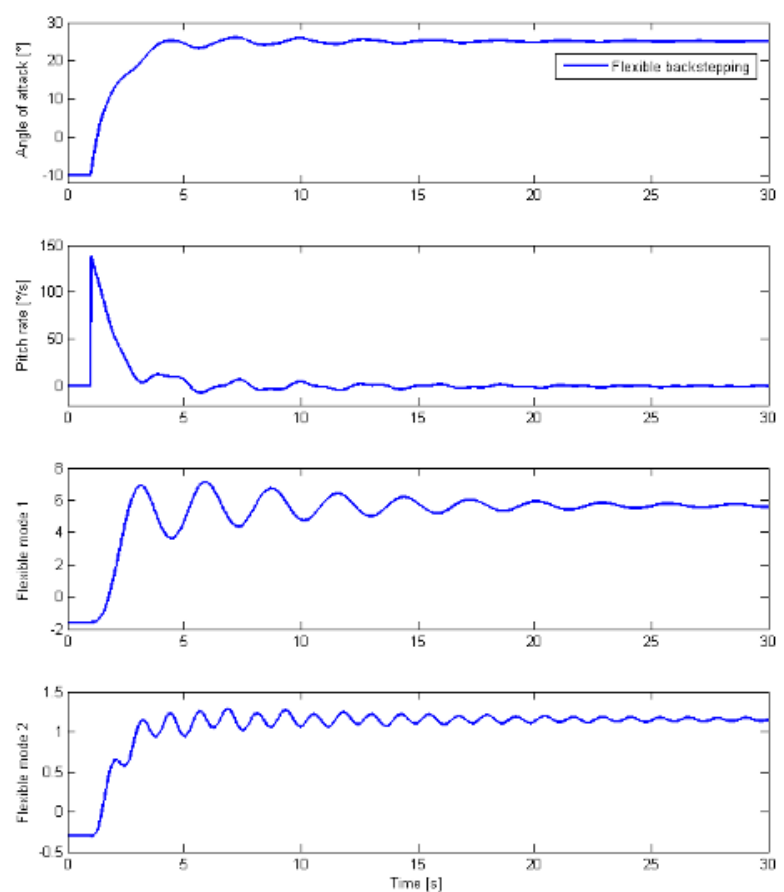

Fig. 1: Evolution of the angle of attack, pitch rate and flexible modes, controlled with the flexible Backstepping law
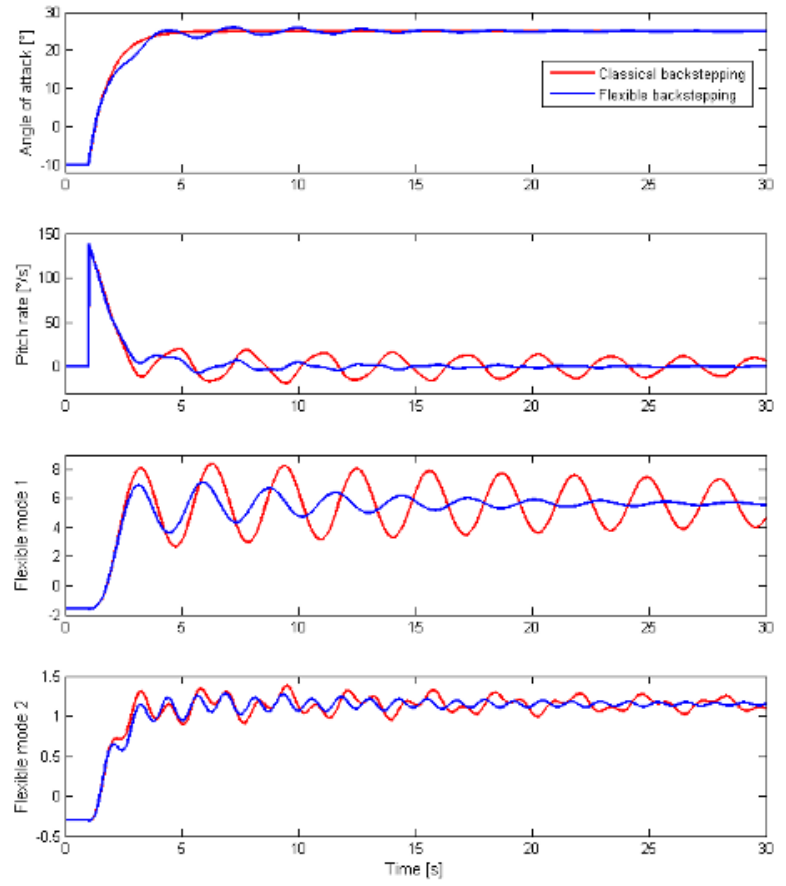

Fig. 2: Limitation of the interaction of the angle of attack dynamics on the transient of the flexible modes 
The evolution of the angle of attack, pitch rate and flexible modes are shown on figure 1. Even if the bending modes are badly damped, the states converge to their equilibria.

We finally make a comparison between the controller we just defined referred to as 'flexible Backstepping' and a classical Backstepping approach using only the rigid-body state. Figure 2 shows that the flexible Backstepping controller enhanced the transient of the flexible modes, as it was noticed in remark 1.

\section{DAMPING OF THE BENDING MODES}

Besides providing the stability of the closed-loop system, we want to suppress the oscillations of the bending modes.

\section{A. Limitation of the control law}

The Lyapunov function $V_{\infty}$ enables to bound the energy of the bending modes. Indeed (22) yields $c_{2} \delta \bar{z}^{T} Q_{z} \delta \bar{z} \leq-\dot{V}_{\infty}$ and by integration one obtains

$$
\int_{0}^{+\infty} \delta \bar{z}^{T} Q_{z} \delta \bar{z} \leq \frac{1}{c_{2}} V_{\infty}(0)
$$

Hence, if $c_{2}$ increases the bending modes can be better damped. However, increasing $c_{2}$ will violate assumption 2 . Moreover tuning $c_{1}$ is useless. Indeed if $\delta \bar{z}(0)=0$ and $\delta q^{*}=0$ we have:

$$
\int_{0}^{+\infty} \delta \bar{z}^{T} Q_{z} \delta \bar{z} \leq \frac{c_{1}}{2 c_{2}} \delta \alpha(0)^{2}
$$
And if we choose $c_{2}=\frac{c_{1} \lambda_{\min }\left(P_{z}\right)}{H_{M}^{2} \lambda_{\max }\left(P_{z}^{2}\right)}(1-\varepsilon)$ with $0<\varepsilon<1$,
we get:

$$
\int_{0}^{+\infty} \delta \bar{z}^{T} Q_{z} \delta \bar{z} \leq \frac{\delta \alpha(0)^{2} H_{M}^{2} \lambda_{\max }\left(P_{z}^{2}\right)}{2 \lambda_{\min }\left(P_{z}\right)(1-\varepsilon)}
$$

This result is independent of $c_{1}$. That means that tuning the control parameters is not sufficient to suppress the bending modes. Figure 3 illustrates this fact. Besides when control parameter $c_{2}$ increases, angle of attack and pitch rate behavior are deteriorated. Thus this control law does not ensure the complete control objective, particularly the damping of the flexible modes. Consequently another method, that consists in changing the local behavior of the trajectories is proposed.

\section{B. Modification of the local behavior}

In this section we use the notation $X$ to designate the state $X=\left[\begin{array}{lll}\alpha & q & z^{T}\end{array}\right]^{T}$

Given a global non-linear controller which guarantees the global stability of an equilibrium, Theorem 3 of [1] gives a sufficient condition to unite this control law with a local optimal one.

Since we have a semi-global non-linear controller, we propose to build a local optimal one to apply this result. The following notations are used for the linearised system:

$$
\delta \dot{X}=A \delta X+B \delta v
$$

where $\delta X=X-X_{c}, \delta v=v-v_{c}, A$ in $\mathbb{R}^{n \times n}$ and $B$ in $\mathbb{R}^{n \times 1}$ are the matrix of the first approximation of system (12).
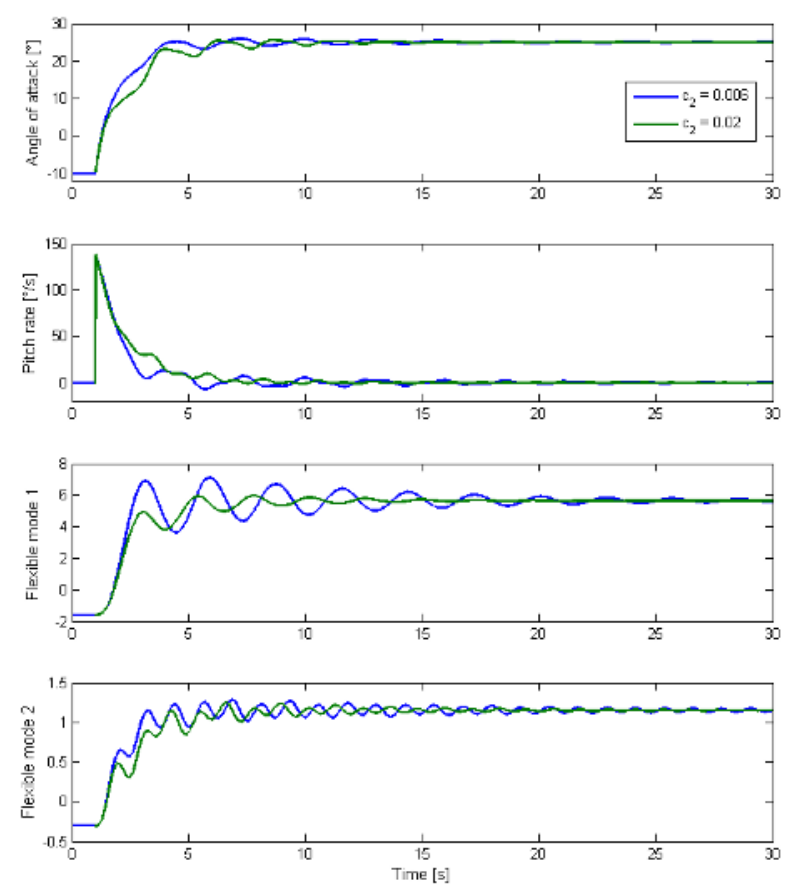

Fig. 3: Influence of control parameter $c_{2}$ on the bending modes
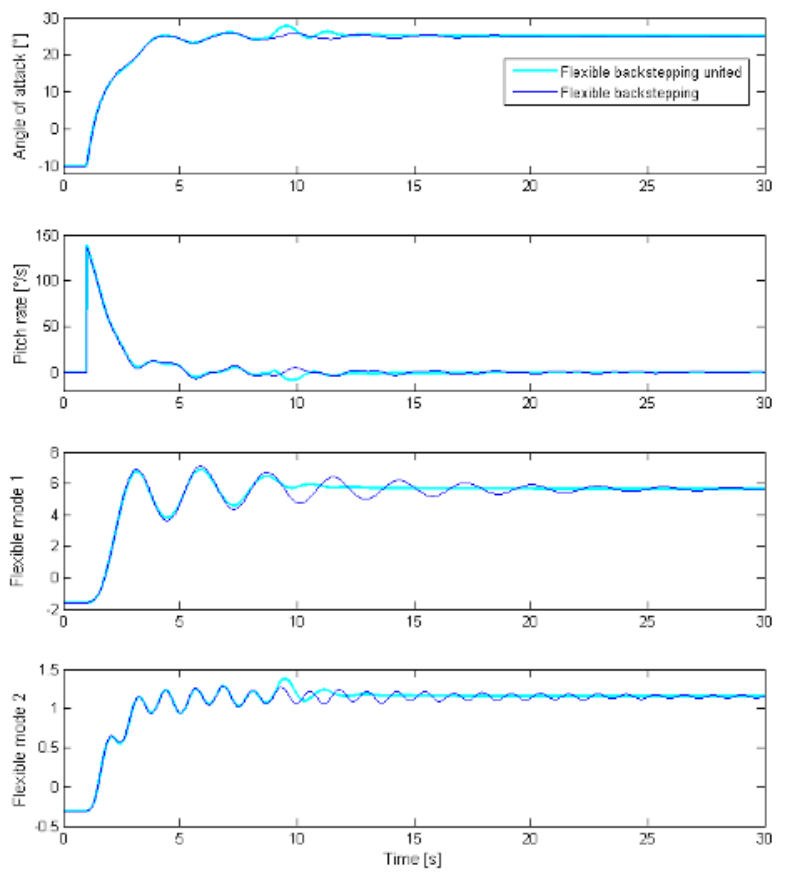

Fig. 4: Modification of the local trajectories with the 'flexible backstepping' controller 
The pair of matrices $(A, B)$ is controllable. We choose to use a LQ controller that makes the following cost as small as possible to minimize the energy of the bending modes:

$$
J=\int_{0}^{t} \delta X^{T} Q \delta X+\delta v^{T} R \delta v
$$

with $Q$ a $n \times n$ matrix and $R$ a positive real. The optimal local controller is given by:

$$
\delta v_{0}=K_{0} \delta X=-\frac{1}{R} B^{T} P_{0} \delta X
$$

where $P_{0}$ in $\mathbb{R}^{n \times n}$ is a symmetric positive definite solution of the associated Ricatti equation $P_{0} A+A^{T} P_{0}-P_{0} B R^{-1} P_{0}=$ $-Q$.

The sufficient condition is formulated as: if there are $K_{m, 1}$ in $\mathbb{R}^{1 \times n}, K_{m, 2}$ in $\mathbb{R}^{1 \times n}$ and $P_{m}$ a definite positive matrix in $\mathbb{R}^{n \times n}$ such that:

$$
\begin{array}{r}
P_{0}\left(A+B K_{m, 1}\right)+\left(A+B K_{m, 1}\right)^{T} P_{0}<0 \\
P_{m}\left(A+B K_{m, 1}\right)+\left(A+B K_{m, 1}\right)^{T} P_{m}<0 \\
P_{m}\left(A+B K_{m, 2}\right)+\left(A+B K_{m, 2}\right)^{T} P_{m}<0 \\
P_{\infty}\left(A+B K_{m, 2}\right)+\left(A+B K_{m, 2}\right)^{T} P_{\infty}<0
\end{array}
$$

where the matrix $P_{\infty}=\frac{\partial^{2} V_{\infty}}{\partial^{2} X}\left(X_{c}\right)$. Then there exists a continuous function $v$ such that the origin of the non-linear system is globally and asymptotically stable and there exists a time from which the local behavior predominates.

We employ the usual linear matrix inequality (LMI) tools to test this sufficient condition through the algorithm:

- given $P_{0}$, solve (28) to get $K_{m_{1}}$,

- given $P_{\infty}$, solve (31) to get $K_{m_{2}}$,

- given $K_{m_{1}}$ and $K_{m_{2}}$, solve (29)-(30) to get $P_{m}$,

Consider the local controller (27), we apply this result to the controller we have designed in this paper, that is the flexible Backstepping. We call 'flexible Backstepping united' the union of these controllers. Figure 4 illustrates this result. The bending modes are better damped. After about 10 seconds the oscillations vanish.

\section{CONCLUSIONS}

In this paper we have presented a non-linear controller design for the longitudinal rotational dynamics of a flexible aircraft. Even if the system is minimum phase, the flexible modes are used by the flight control law in order to obtain a better transient of the full state of the system. Moreover, the local behavior of the trajectories have been modified to achieve the control objectives particularly to damp out the bending modes.

Let us also remark that we have illustrated our results on a 2flexible modes model although there is no obstacle to apply them to a similar system with a large number of flexible modes.

In the future, the entire longitudinal dynamics of a flexible aircraft will be considered and a non-linear observer will be added to address the output Feedback problem since the flexible modes are usually not measured.

\section{REFERENCES}

[1] M. Sahnoun, V. Andrieu and M.Nadri, Nonlinear and locally optimal controllers design for input affine systems, International Journal of Control, Vol 85, Issue 2, Pages 159-170, February 2012.

[2] L. Fiorentini, A. Serrani, M. Bolender and D. Doman, Nonlinear Robust Adaptative Control of Flexible Air-Breathing Hypersonic Vehicles, Journal of Guidance, Control and Dynamics, vol. 32, no. 2, pp. 402-417, 2009.

[3] M. Reyhanoglu and J. Hervas, Point-to-Point Liquid Container Transfer via a PPR Robot with Sloshing Suppression, in Proc. American Control Conference, Montréal, pp. 5490-5494, 2012.

[4] M. Reyhanoglu and J. Hervas, Nonlinear dynamics and control of space vehicles with multiple fuel slosh modes, Control Engineering Practice, vol. 20, pp. 912-918, 2012.

[5] M. Bolender and D. Doman, Nonlinear Longitudinal Dynamical Model of an Air-Breahting Hypersonic Vehicle, Journal of Spacecraft and Rockets, vol.44, no. 2, pp. 374, 2007.

[6] A. Isidori, Nonlinear Control Systems , Springer, third edition, 1995.

[7] R. Sepulchre, M. Jankovic and P.V. Kokotovic, Constructive nonlinear control, Springer, 1997.

[8] R. Wood and B. Cazzolato, An Alternative Nonlinear Control Law for the Global Stabilization of the PVTOL Vehicle, IEEE Transactions on Automatic Control, vol.52, no. 7, pp. 1282-1287, 2007.

[9] R. Olfati-Saber, Nonlinear Control of Underactuated Mechanical Systems with Application to Robotics and Aerospace Vehicles, PHD Thesis, MIT, Cambridge, MA, February 2001.

[10] S. Nazrulla and H.K. Khalil, Robust Stabilization of Non-Minimum Phase Nonlinear Systems Using Extended High-Gain Observers, IEEE Transactions on Automatic Control, vol.56, no. 4, pp. 802-813, 2011.

[11] A. Astolfi, New Results on the Global Stabilization of Minimum-Phase Nonlinear Systems, Automatica, vol.34, no. 6, pp. 783-788, 1998.

[12] I. Mizumoto and R. Kohzawa, Control of a Flexible Arm with Input Dead Zone by a Passivity based Adaptive Output Feedback, IEEE International Conference on Control Applications, Dubrovnic, Croatia ,pp. 533-538, 2012.

[13] S. Su and Y. Lin, Robust output tracking control of a class of nonminimum phase systems and application to VTOL aircraft, International Journal of Control, vol.84, no. 11, pp. 1858-1872, 2011. 